\title{
On wave functional in QED
}

\author{
Daqing Liu \\ Department of Applied Physics, Xi'an Jiaotong University, P.R. China
}

\begin{abstract}
In a discrete form of the second quantization, the gauge independencies of all the physical states including vacuum in QED are restudied through a new approach. We also discuss an interesting phenomenon attributed to vacuum effect and come up with a procedure to produce general physical states.
\end{abstract}

PACS: 03.65.Db, 12.70.Ds

\section{Introduction}

Quantization of electromagnetic field theory is a textbook task ${ }^{[1]}$. However, quantization using canonical formulation faces two conflicting requirements: 1) the theory is gauge invariant; 2) there is lower bound to the free field energy. A obvious result from the confliction is that we always introduce ghost states in canonical quantization, such as gauge-dependent temporal photon and longitude photon. As is known, one way to exclude the ghost states is to take gauge fixing, for example, Coulomb gauge. However, under gauge fixings, the theory always loses gauge independence. Furthermore, in temporal gauge, the gauge field is not fixed and ghost states are still needed in canonical quantization.

Advanced studies show that there is possibly another way to exclude ghost states, functional approach ${ }^{[2]}$. For references we refer to [1-7] etc. Refs. $[2,5,6]$ are on fermions while Refs. $[1,3,4]$ on the ground state of gauge fields. However, in the functional approach, the same as in the canonical quantization, the gauge independence of physical states is not very obvious. Lee argues that all states should be gauge independent to consist with a peculiar phenomenon, color confinement, in QCD ${ }^{[7]}$. But such phenomenon does not occur in QED. Here we propose a new approach which can ensure that all physical states in QED should also be gauge independent.

This approach takes advantage of the fact that QED possesses an expansion symmetry in gauge space, which is the generalization of local gauge transformation. Since such symmetry is not held for general wave functionals, it is natural to require that the energy of physical wave functional is invariant under such transformation. Such requirement leads to the gauge independence of wave functional in QED.

To avoid divergences and ambiguities in continuous theory attributed to infinite ultraviolet and infrared cut- off, we discretize the position space by dividing the box with size $L^{3}$ into $N(\rightarrow \infty)$ grids with spacing $\Delta x=\Delta y=$ $\Delta z=\frac{L}{N^{1 / 3}}$ to get a finite ultraviolet and infrared cutoff. For instance, if we set $L \rightarrow \infty$, we shall obtain divergent results in Eqs. (21), etc. Furthermore, if space-time is indeed discrete and/or QED is invalid beyond some energy scale, the discreteness will have physical meaning.

In section 2 we list results of quantization to free QED. We show that under a reasonable assumption, all the physical states are gauge independent in section 3 . Section 4 studies state functional including vacuum in detail. Section 5 is a simple discussion.

\section{The Quantization to free QED}

This section shows the main results of the quantization to free QED briefly. For simplicity, we set $A_{0} \equiv 0$.

The commutation relations of gauge fields $A_{i}(\mathbf{x})$ and adjoint fields $\Pi_{i}(\mathbf{x})$ read

$$
\left[A_{i}(\mathbf{x}), \Pi_{j}\left(\mathbf{x}^{\prime}\right)\right]=\frac{\mathrm{i}}{\tau} \delta_{i j} \delta_{\mathbf{x}, \mathbf{x}^{\prime}},
$$

where $\tau=\Delta x^{3}$. In other words, $\Pi_{i}(\mathbf{x})=-\mathrm{i} \frac{\partial}{\tau \partial A_{i}(\mathbf{x})}$. Meanwhile, suppose Fourier transformations of gauge fields and their conjugate fields are defined as

$$
A_{i}(\mathbf{p})=\sum \tau A_{i}(\mathbf{x}) e^{-i \mathbf{p} \cdot \mathbf{x}}, \Pi_{i}(\mathbf{p})=\sum \tau \Pi_{i}(\mathbf{x}) e^{i \mathbf{p} \cdot \mathbf{x}}
$$

respectively, then

$$
\left[A_{i}(\mathbf{p}), \Pi_{j}\left(\mathbf{p}^{\prime}\right)\right]=\left[A_{i}^{*}(\mathbf{p}), \Pi_{j}^{*}\left(\mathbf{p}^{\prime}\right)\right]=\mathrm{i} L^{3} \delta_{i j} \delta_{\mathbf{p} \mathbf{p}^{\prime}} .
$$

We also introduce the magnetic fields $B_{i}(\mathbf{x})=$ $\epsilon_{i j k} \partial_{j} A_{k}(\mathbf{x})$, or, $B_{i}(\mathbf{p})=\mathrm{i} \epsilon_{i j k} \hat{p_{j}} A_{k}(\mathbf{p})$, where $B_{i}(\mathbf{p})=$ $\sum \tau B_{i}(\mathbf{x}) e^{-i \mathbf{p} \cdot \mathbf{x}}$ and $\hat{p}_{j} \equiv \frac{1}{\Delta x} \sin p_{j} \Delta x$ (thereinafter we 
always ignore the hat symbol without confusion). Thus, for instance, with the notation $\frac{\Delta \mathbf{p}^{3}}{(2 \pi)^{3}}=1 / L^{3}$,

$$
\frac{\partial}{\tau \partial A_{i}(\mathbf{x})}=\mathrm{i} \epsilon_{i j k} L^{3} \sum_{\mathbf{p}} \frac{\Delta \mathbf{p}^{3}}{(2 \pi)^{3}} e^{-i \mathbf{p} \cdot \mathbf{x}} p_{j} \frac{\partial}{\partial B_{k}(\mathbf{p})} .
$$

Since $B_{i}(\mathbf{x})$ and $A_{i}(\mathbf{x})$ are both real, state functionals are invariant under transformation $A_{i}(\mathbf{p}) \rightarrow A_{i}^{*}(-\mathbf{p})$ or $B_{i}(\mathbf{p}) \rightarrow B_{i}^{*}(-\mathbf{p})$.
We read Hamiltonian as ${ }^{[7]}$

$$
\begin{aligned}
H & =\frac{1}{2} \sum_{x} \tau\left[\Pi_{i} \Pi_{i}^{*}+B_{i} B_{i}^{*}\right] \\
& =\frac{1}{2} \sum_{x} \tau\left[-\frac{\partial^{2}}{\Delta x^{6} \partial A_{i} \partial A_{i}^{*}}+B_{i} B_{i}^{*}\right] .
\end{aligned}
$$

Or, in Fourier space,

$$
\begin{aligned}
H & =\frac{1}{2} \sum \frac{\Delta \mathbf{p}^{3}}{(2 \pi)^{3}}\left\{-L^{6} \frac{\partial^{2}}{\partial A_{i}(\mathbf{p}) \partial A_{i}^{*}(\mathbf{p})}+p^{2} A_{i}(\mathbf{p}) A_{i}^{*}(\mathbf{p})-p_{i} A_{i}(\mathbf{p}) p_{j} A_{j}^{*}(\mathbf{p})\right\} \\
& =\frac{1}{2} \sum \frac{\Delta \mathbf{p}^{3}}{(2 \pi)^{3}}\left\{-L^{6} \frac{p^{2} \partial^{2}}{\partial B_{k}(\mathbf{p}) \partial B_{k}^{*}(\mathbf{p})}+L^{6} \frac{p_{j} p_{k} \partial^{2}}{\partial B_{k}(\mathbf{p}) \partial B_{j}^{*}(\mathbf{p})}+B_{i}(\mathbf{p}) B_{i}^{*}(\mathbf{p})\right\} .
\end{aligned}
$$

\section{Gauge independence of state solutions $\Theta=\prod_{\mathbf{p}} \Theta_{\mathbf{p}}[\mathbf{A}(\mathbf{p})]$, where $\Theta_{\mathbf{p}}$ 's satisfy
functionals}

We show here the properties of state functionals under gauge transformation. Hamiltonian in equation (7) can be divided into $H=\sum_{\mathbf{p}} H_{\mathbf{p}}$, where

$$
\begin{aligned}
H_{\mathbf{p}}= & \frac{1}{2 L^{3}}\left\{-L^{6} \frac{\partial^{2}}{\partial A_{i}(\mathbf{p}) \partial A_{i}^{*}(\mathbf{p})}+p^{2} A_{i}(\mathbf{p}) A_{i}^{*}(\mathbf{p})-\right. \\
& \left.p_{i} A_{i}(\mathbf{p}) p_{j} A_{j}^{*}(\mathbf{p})\right\} .
\end{aligned}
$$

Therefore, equation $H \Theta=E \Theta$ possesses separable

$$
\begin{array}{r}
\left\{-L^{6} \frac{\partial^{2}}{\partial A_{i}(\mathbf{p}) \partial A_{i}^{*}(\mathbf{p})}+p^{2} A_{i}(\mathbf{p}) A_{i}^{*}(\mathbf{p})-\right. \\
\left.p_{i} A_{i}(\mathbf{p}) p_{j} A_{j}^{*}(\mathbf{p})\right\} \Theta_{\mathbf{p}}=2 E_{\mathbf{p}} L^{3} \Theta_{\mathbf{p}},
\end{array}
$$

with the total energy $E=\sum_{\mathbf{p}} \frac{\Delta \mathbf{p}^{3}}{(2 \pi)^{3}} E_{\mathbf{p}} L^{3}=\sum E_{\mathbf{p}}$.

As for a definite $\mathbf{p}$, the theory is rotation invariance providing $p \ll \Delta x^{-1}$. One can, therefore, rotate vector $\mathbf{p}$ into $\mathbf{p}_{0}=(0,0, p)$. For such $\mathbf{p}_{0}$ we get

$$
\left\{-L^{6} \sum_{i} \frac{\partial^{2}}{\partial A_{i}\left(\mathbf{p}_{0}\right) \partial A_{i}^{*}\left(\mathbf{p}_{0}\right)}+p^{2} A_{1}\left(\mathbf{p}_{0}\right) A_{1}^{*}\left(\mathbf{p}_{0}\right)+A_{2}\left(\mathbf{p}_{0}\right) A_{2}^{*}\left(\mathbf{p}_{0}\right)\right\} \Theta_{\mathbf{p}_{0}}=2 E_{\mathbf{p}_{0}} L^{3} \Theta_{\mathbf{p}_{0}} .
$$

Eq. (10) also possesses separable solution $\Theta_{\mathbf{p}_{0}}=X\left[A_{1}\left(\mathbf{p}_{0}\right), A_{1}\left(-\mathbf{p}_{0}\right)\right] Y\left[A_{2}\left(\mathbf{p}_{0}\right), A_{2}\left(-\mathbf{p}_{0}\right)\right] Z\left[A_{3}\left(\mathbf{p}_{0}\right), A_{3}\left(-\mathbf{p}_{0}\right)\right]$, with $X, Y, Z$ satisfying

$$
\left\{\begin{array}{c}
\left\{-L^{6} \frac{\partial^{2}}{\partial A_{1}\left(\mathbf{p}_{0}\right) \partial A_{1}^{*}\left(\mathbf{p}_{0}\right)}+p^{2} A_{1}\left(\mathbf{p}_{0}\right) A_{1}^{*}\left(\mathbf{p}_{0}\right)\right\} X=2 E^{X} L^{3} X, \\
\left\{-L^{6} \frac{\partial^{2}}{\partial A_{2}\left(\mathbf{p}_{0}\right) \partial A_{2}^{*}\left(\mathbf{p}_{0}\right)}+p^{2} A_{2}\left(\mathbf{p}_{0}\right) A_{2}^{*}\left(\mathbf{p}_{0}\right)\right\} Y=2 E^{Y} L^{3} Y, \\
-L^{6} \frac{\partial^{2}}{\partial A_{3}\left(\mathbf{p}_{0}\right) \partial A_{3}^{*}\left(\mathbf{p}_{0}\right)} Z=2 E^{Z} L^{3} Z,
\end{array}\right.
$$

where $E_{\mathbf{p}_{0}}=E^{X}+E^{Y}+E^{Z}$.

Now we have divided $\Theta_{\mathbf{p}_{0}}$ into two parts. One of them, $X$ and $Y$, is perpendicular to gauge transformation, and the other, $Z$, is parallel to gauge transformation. The perpendicular part resembles harmonic oscillator while parallel part free particle.

As for physical state, $X(Y, Z)$ tends to zero when $\left|A_{i}\right| \rightarrow \infty(i=1,2,3)$. For $X$ and $Y$, with analogy to oscillator, there is no problem. But for $Z$, there is no solution satisfying the condition. Up to a constant, the general solution can be written as $Z=$ $\exp \left\{a A_{3}-2 E^{Z} L^{-3} a^{-1} A_{3}^{*}\right\}$, where, for simplicity, $A_{3}$ and $A_{3}^{*}$ stand for $A_{3}\left(\mathbf{p}_{0}\right)$ and $A_{3}^{*}\left(\mathbf{p}_{0}\right)$ respectively. But this solution is not convergent when $\left|A_{3}\right| \rightarrow \infty$, provided $a a^{*} \neq 2 E^{Z} L^{-3}$. States with $E^{Z}<0$ can also be ruled out by the divergence of functional at $\left|A_{3}\right| \rightarrow \infty$. Meanwhile, the choice of $|a|=\sqrt{2 E^{Z} L^{-3}}\left(E^{Z} \geq 0\right)$ gives a 
finite but non-vanishing $Z$ when $\left|A_{3}\right| \rightarrow \infty$. Since each eigen-functional, including for $E^{Z}=0$, has such problem, we take a modified constraint on $Z: Z$ is finite when $\left|A_{3}\right| \rightarrow \infty$.

Thus we obtain $Z=e^{a A_{3}-a^{*} A_{3}^{*}}$ with $|a|=\sqrt{2 E^{Z} L^{-3}}$. Here $A_{3}$ or $\mathbf{p} \cdot \mathbf{A}$ is free completely, correspondingly, $\Pi_{3}$ or $\mathbf{p} \cdot \boldsymbol{\Pi}$ is determined absolutely, which can also be seen from the conservation of $\mathbf{p} \cdot \boldsymbol{\Pi},[\mathbf{p} \cdot \boldsymbol{\Pi}, H] \equiv 0$. This is a special case of Heisenberg Uncertainty Principle.

It easy to see from Eq. (5) that the system has symmetry, $A_{i} \rightarrow A_{i}-p_{i} f, \Pi_{i} \rightarrow \Pi_{i}$. The local gauge symmetry corresponds to a translation in gauge space, since $f$ is an arbitrary scalar function of $\mathbf{p}$. However, $f$ can also be a scalar function with respect to gauge fields, for instance, $f=p_{i} A_{i} \epsilon$, where $\epsilon$ is independent of $A_{i} 1$ It is easy to check that under this transformation $\boldsymbol{\Pi}$ and $\mathbf{B}$ remain unchanged. Therefore, besides local gauge symmetry, QED also possesses an expansion symmetry in gauge space.

However, unlike the local gauge symmetry, the expansion symmetry is broken after Eq. (6). To see it we perform a transformation in gauge space, $\mathbf{A} \rightarrow \mathbf{A}+\mathbf{p} h$, where we choose scalar function $h=\epsilon \mathbf{p} \cdot \mathbf{A} / p^{2}$. At $\mathbf{p}=\mathbf{p}_{0}$ we get

$$
\left\{\begin{array}{c}
A_{3} \rightarrow A_{3}+a \epsilon A_{3}, \\
A_{3}^{*} \rightarrow A_{3}^{*}+a^{*} \epsilon^{*} A_{3}^{*}, \\
A_{1}\left(A_{2}, A_{1}^{*}, A_{2}^{*}\right)(\mathbf{p}) \rightarrow A_{1}\left(A_{2}, A_{1}^{*}, A_{2}^{*}\right)(\mathbf{p}),
\end{array}\right.
$$

Then in equation (11) $Z \rightarrow Z^{\prime}=e^{a(1+\epsilon) A_{3}-a^{*}\left(1+\epsilon^{*}\right) A_{3}^{*}}$. The new functional has a changed energy, $E^{\prime Z}=\mid 1+$ $\left.\epsilon\right|^{2} E^{Z}$, that is, the state has a energy of gauge dependence as long as $E^{Z} \neq 0$. The statement can be considered in another way. As we know, $Z$ is a functional with a (complex) period, which is in proportion to $\left(E^{Z}\right)^{-1}$ up to a phase factor. Since the above transformation changes the period of the functional, it can also change $E^{Z}$.

We are faced with a puzzle: On one hand, $E^{Z}$ is a conservational quantity, while on the other hand, it can be changed by an unphysical expansion in gauge space. To treat this puzzle, reference [9] makes a gauge fixing, such as $A_{3}=0$, and no $\Pi_{3}$ existing correspondingly, for it is thought that neither $A_{3}$ nor $\Pi_{3}$ has physical meaning, or, in other words, they are both redundant variables at the case of $\mathbf{p}=\mathbf{p}_{0}$. This treatment takes gauge dependent functionals and one should also modify the commutation relation (3). Here we can treat it in another way. We do not take the gauge fixing and therefore do not change the commutation relation. On the contrary, we think that all the physical states have a natural constraint: the energy of physical state does not change under the gauge translations and gauge expansions, since these transformations are both unphysical. This requirement will lead to $E^{Z}=0$ and therefore $Z \equiv 1$. Therefore, although there is no phenomenon similar to color confinement, all the states should be gauge invariant in QED. For general p, the statement can be written as

$$
p_{i} \Pi_{i} \Theta=p_{i} \Pi_{i}^{*} \Theta=0 .
$$

The puzzle nominated as color confinement in QCD has been treated by many researches, most of which are based on some combined forces. For instance, in reference [10] the author introduces a non-local Coulomb interactions between color charge. Here we show a somewhat different viewpoint.

In non-Abelian case, especially $S U(3)$ theory or QCD, we face a very tanglesome situation. In QED, interactions in Hamiltonian is local in Fourier space (up to $\mathrm{a} \pm \mathbf{p})$. However, they are nonlocal in non-Abelian theory. This is because there occur cubic and quartic interactions in QCD. An infinitesimal local gauge transformation (in position space) connects different momentum and color direction(A finite local gauge transformation even connects states with different numbers of gluons). Consider a gluon with single momentum (up to a $\pm \mathbf{p}$ ) and/or single color direction. Suppose it is an eigen-state of Hamiltonian in QCD, it can be written as $B_{i}^{a}(\mathbf{p}) \Theta_{0}$ (or $A_{i}^{a}(\mathbf{p}) \Theta_{0}$ ), where superscript and subscript are color index and direction index respectively. The gluon will be connected with other gluons with different momentum and/or directions, for instance, $B_{i}^{b}\left(\mathbf{p}^{\prime}\right) \Theta_{0}$ (Generally, $\left.\left|\mathbf{p}^{\prime}\right| \neq|\mathbf{p}|\right)$ by local gauge transformation, for the QCD vacuum $\Theta_{0}$ is gauge invariant( This is a significant difference between Abelian theory and non-Abelian theory). Therefore, since $B_{i}^{a}(\mathbf{p}) \Theta_{0}$ and $B_{i}^{b}\left(\mathbf{p}^{\prime}\right) \Theta_{0}$ are connected by a local gauge transformation, which does not change state energy, they have the same energy. This is impossible unless single gluon with definite momentum is infinite heavy. Or, a single gluon is eigenstate of Hamiltonian if and only if it is infinite heavy.

Unlike in reference [9], our treatment keeps the commutation relations in equation (3) unchanged and does not introduce gauge condition. By a constraint on physical states, we find that, attributed to the gauge expansion symmetry, not only vacuum, but also all the physical states are gauge independent.

\section{Solution to state functional}

In this section we show the solution to general wave functional. First we review the functional of vacuum, the

1 This corresponds to a transformation $A_{i}(\mathbf{x}) \rightarrow A_{i}(\mathbf{x})+\sum \Delta y^{3} \frac{\partial A_{j}(\mathbf{y})}{\partial y_{j}} \frac{\partial h(\mathbf{x}-\mathbf{y})}{\partial x_{i}}$. The transformation is not local in position space, but in Fourier space, it is. 
eigen-functional with the lowest energy.

At first, one possibly prefers writing the vacuum state as functional with respect to $B_{i}$. But such treatment will meet a singularity. To see it we write the vacuum state from equation (9) as

$$
\Theta_{0}=\exp \left\{-\sum \frac{\Delta \mathbf{p}^{3}}{(2 \pi)^{3}} B_{i}^{*}(\mathbf{p}) D_{i k}^{0}(\mathbf{p}) B_{k}(\mathbf{p})\right\},
$$

due to the translation invariance. Introducing a positive matrix $S^{0}(\mathbf{p})=D^{0}(\mathbf{p})+D^{0 T}(-\mathbf{p})$ we have

$$
1 / p^{2}=S^{0}\left(1-\bar{P} / p^{2}\right) D^{0},
$$

where $(\bar{P})_{m n}=p_{m} p_{n}$. There is no solution to this equation, for the determinant of l.h.s. equal to $\left(1 / p^{2}\right)^{3}$ while the determinant of r.h.s. equal to zero, unless the determinant of matrix $S^{0}$ equals to infinity.

To see it more clearly, we write $S^{0}=\frac{1}{p}\left(1-\bar{P} / p^{2}\right)^{-1 / 2}$ naively. Suppose $\mathbf{p}=\left(0,0, p_{3}\right)$, or $1-\bar{P} / p^{2}=$ $\operatorname{diag}(1,1,0)$, we then obtain a singular $S_{33}^{0}$. This reveals an obvious fact that there is no longitudinal magnetic fields in free QED.

Therefore, a more convenient proposal is to write the vacuum state as functional with respect to $A_{i}$,

$$
\Theta_{0}=\exp \left\{-\sum_{\mathbf{p}} \frac{\Delta \mathbf{p}^{3}}{(2 \pi)^{3}} A_{i}^{*}(\mathbf{p}) D_{i k}(\mathbf{p}) A_{k}(\mathbf{p})\right\} .
$$

Repeating the deductions, we obtain,

$$
D=\frac{p}{2}\left(1-\bar{P} / p^{2}\right)
$$

It is easy to check that $\Theta_{0}[\mathbf{A}(\mathbf{p})]=\Theta_{0}[\mathbf{A}(\mathbf{p})+\mathbf{p} h]$, where $h$ is an arbitrary scalar function. As expected, $\Theta_{0}$ is gauge independent.

Iterate $B_{i}(\mathbf{p})=\mathrm{i} \epsilon_{i j k} p_{j} A_{k}(\mathbf{p})$ into Eq. (16), we write the vacuum functional as,

$$
\Theta_{0}=\exp \left\{-\sum \frac{\Delta \mathbf{p}^{3}}{(2 \pi)^{3}} \frac{1}{2 p} B_{i}^{*}(\mathbf{p}) B_{i}(\mathbf{p})\right\},
$$

with a constraint $p_{i} B_{i}=p_{i} B_{i}^{*}=0$. This result is in agreement with the references [3,4], except a necessary constraint. Since canonical fields are $\mathbf{A}(\mathbf{x})$, we prefer (16) to (18) as our final result.

For the density of the ground state energy, we have

$$
\mathcal{E}_{0}=E_{0} / L^{3}=\frac{1}{2} \sum \frac{\Delta \mathbf{p}^{3}}{(2 \pi)^{3}} 2 D_{i i}(\mathbf{p})=\frac{d}{2} \sum \frac{\Delta \mathbf{p}^{3}}{(2 \pi)^{3}} p,
$$

where $d=3-1=2$ is just the degree of freedom. Thus, due to the gauge invariance, the degree of freedom is not three but two for each $\mathbf{p}$. In discrete form $\mathcal{E}_{0}$ is

$$
\int_{\frac{-\pi}{\Delta x}}^{\frac{\pi}{\Delta x}} \frac{\Delta \mathbf{p}^{3}}{(2 \pi)^{3}} \sqrt{\sin ^{2} p_{x} \Delta x+\sin ^{2} p_{y} \Delta x+\sin ^{2} p_{z} \Delta x} \simeq \frac{1.19}{\tau} .
$$

The ultraviolet cutoff in the Fourier space is just the inverse size of grids $\Delta x^{-1}$. In continuous l.h.s. in the above equation should be $\int_{\frac{-\pi}{\Delta x}}^{\frac{\pi}{\Delta x}} \frac{\Delta \mathbf{p}^{3}}{(2 \pi)^{3}} p$, which is about 630 times larger than the discrete one. It is significant that the zero-point energy in the discrete form is much lower than that in the continuous form.

The most possible measurements of canonical fields and their conjugate fields, electric fields, are vanishing at each p. However, other measurements are still possible. This is uncertainty in quantum mechanism. In fact, any definite configuration, such as $\mathbf{A}_{i}(\mathbf{x}) \equiv 0$, is never the eigenstate of Hamiltonian, attributed to the uncertainty. Thus, if we put an electric dipole in a box, its motion will be changed by nonzero electric field originated from the uncertainty. Such effect is suppressed by the volume of box.

The uncertainty also leads to the condensates of the gauge fields. Without loss of generality we set $\mathbf{p}_{0}=$ $\left(0,0, p_{3}\right)$. We have now $<A_{3}^{*}\left(\mathbf{p}_{0}\right) A_{3}\left(\mathbf{p}_{0}\right)>=\infty$ for the vacuum is gauge independent. But, the condensates $<A_{1}^{*}\left(\mathbf{p}_{0}\right) A_{1}\left(\mathbf{p}_{0}\right)>=<A_{2}^{*}\left(\mathbf{p}_{0}\right) A_{2}\left(\mathbf{p}_{0}\right)>$ are finite:

$$
\begin{aligned}
& <A_{1}^{*}\left(\mathbf{p}_{0}\right) A_{1}\left(\mathbf{p}_{0}\right)>=\frac{\int[d \mathbf{A}(\mathbf{p})] A_{1}^{*}\left(\mathbf{p}_{0}\right) A_{1}\left(\mathbf{p}_{0}\right) \Theta_{0}^{2}}{\int[d \mathbf{A}(\mathbf{p})] \Theta_{0}^{2}} \\
& =\frac{\int d A_{1}\left(\mathbf{p}_{0}\right) A_{1}^{*}\left(\mathbf{p}_{0}\right) A_{1}\left(\mathbf{p}_{0}\right) \exp \left\{-\frac{2 p_{0}}{L^{3}} A_{1}^{*}\left(\mathbf{p}_{0}\right) A_{1}\left(\mathbf{p}_{0}\right)\right\}}{\int d A_{1}\left(\mathbf{p}_{0}\right) \exp \left\{-\frac{2 p_{0}}{L^{3}} A_{1}^{*}\left(\mathbf{p}_{0}\right) A_{1}\left(\mathbf{p}_{0}\right)\right\}} \\
& =\frac{L^{3}}{2 p_{0}},
\end{aligned}
$$

where $p_{0}=\left|p_{3}\right|$.

One can furthermore obtain the gauge independent condensates,

$$
\begin{aligned}
& <B_{i}^{*}\left(\mathbf{p}_{0}\right) B_{i}\left(\mathbf{p}_{0}\right)>= \\
& p_{0}^{2}<A_{1}\left(\mathbf{p}_{0}\right)^{*} A_{1}\left(\mathbf{p}_{0}\right)+A_{2}^{*}\left(\mathbf{p}_{0}\right) A_{2}\left(\mathbf{p}_{0}\right)>=\frac{2}{2} p_{0} L^{3}, \\
& <\Pi_{i}^{*}\left(\mathbf{p}_{0}\right) \Pi_{i}\left(\mathbf{p}_{0}\right)>= \\
& -L^{6}<\frac{\partial^{2}}{\partial A_{i}^{*}\left(\mathbf{p}_{0}\right) \partial A_{i}\left(\mathbf{p}_{0}\right)}>=\frac{2}{2} p_{0} L^{3} .
\end{aligned}
$$

The gauge invariance implies $\Pi_{3}\left(\mathbf{p}_{0}\right)=0$ in the vacuum with a completely free $A_{3}\left(\mathbf{p}_{0}\right)$. Notice here that not only gauge field but also gauge potential, which is gauge dependent, has gauge independent expectations. One can generalize Eq. (22) to general $\mathbf{p}$ in a straight way, which leads to an expected result, $E_{0}=\frac{1}{2} \sum \frac{\Delta \mathbf{p}^{3}}{(2 \pi)^{3}}\{<$ $\left.B_{i}^{*}(\mathbf{p}) B_{i}(\mathbf{p})>+<\Pi_{i}^{*}(\mathbf{p}) \Pi_{i}(\mathbf{p})>\right\}$.

It is also interesting to study correlators of the gauge 
fields at different positions. The results are

$$
\begin{aligned}
<B_{i}^{*}(\mathbf{x}) B_{i}(0)> & =\frac{1}{L^{3}} \sum \frac{\Delta \mathbf{p}^{3}}{(2 \pi)^{3}}<B_{i}(\mathbf{p}) B_{i}^{*}(\mathbf{p})>e^{i \mathbf{p}} \\
& =\sum \frac{\Delta \mathbf{p}^{3}}{(2 \pi)^{3}} p e^{i \mathbf{p} \cdot \mathbf{x}} \\
<\Pi_{i}^{*}(\mathbf{x}) \Pi_{i}(0)> & =\sum \frac{\Delta \mathbf{p}^{3}}{(2 \pi)^{3}} p e^{i \mathbf{p} \cdot \mathbf{x}} .
\end{aligned}
$$

The study shows that the vacuum proposes many properties similar to that of the ground state in the harmonic oscillator, but there are also some different properties, due to the gauge invariance of QED vacuum. Because of the quantum effect, or, uncertainty, the vacuum has a complex structure, for instance, the nonvanishing condensates and correlate.

The following is a simple study on the general solutions. We emphasize again that all the state must be gauge invariant. We take solution to Eq. (9).

Setting $A_{i}^{\perp} \equiv A_{j}(\mathbf{p})\left(\delta_{i j}-p_{i} p_{j} / p_{0}^{2}\right), \quad \Theta_{\mathbf{p}_{0}} \equiv$ $\Theta_{\mathbf{p}_{0}}\left[A_{i}^{\perp}, A_{i}^{\perp *}\right]=f \Theta_{0 \mathbf{p}_{0}}$ and $\Theta_{0 \mathbf{p}_{0}}=\exp \left\{-\frac{p_{0}}{L^{3}} A_{i}^{\perp} A_{i}^{\perp *}\right\}$ with function $f$ to be determined, we have,

$$
\begin{aligned}
E_{\mathbf{p}_{0}} f= & -L^{3}\left(\delta_{i j}-p_{i} p_{j} / p_{0}^{2}\right) \frac{\partial^{2} f}{\partial A_{i}^{\perp *} \partial A_{j}^{\perp}}+ \\
& p_{0}\left(\frac{\partial f}{\partial A_{i}^{\perp}} A_{i}^{\perp}+\frac{\partial f}{\partial A_{i}^{\perp *}} A_{i}^{\perp *}\right),
\end{aligned}
$$

where we have ignored the ground state energy.

One can use equation (24) to study states of photons. For instance, up to a constant, we obtain a quantum state

$$
\Theta^{k}=\left(c_{k} A_{k}^{\perp}+c_{k}^{*} A_{k}^{\perp *}\right) \Theta_{0},
$$

where $c_{k}=e^{i \theta_{k}}$ and $\Theta_{0}=\prod_{\text {pairs of } \mathbf{p}} \Theta_{0 \mathbf{p}}$ is the vacuum.

Notice here $p_{k} \Theta^{k} \equiv 0$. It is not difficult to verify that $\Theta^{k}$ 's are two eigenstates with linear polarization perpendicular to the momentum $\mathbf{p}_{0}$.

One can use the skill of state superposition to construct the states of photon corresponding to other direction of linear polarization or corresponding to circular polarization. Furthermore, the study of state of single photon can also be generalized to other states, for instance, states of multi-photon.

\section{Discussions}

The gauge independencies of QED are studied through a new approach, which is related to a generalization of the local gauge symmetry, the expansibility in gauge space. The study shows clearly that all the physical states are gauge independent.

Our study reveals clearly why there are just two degrees of freedom in gauge field and therefore the introduction of ghost states is not needed. Furthermore, we show that not only all physical states, but also expectations of operators, some of which, for instance, gauge potential, $A_{i}$, is not gauge independent, should be gauge invariant.

All the states should be gauge invariant both in QED and in QCD. Whereas, there is a crucial difference between QED and QCD, that is, gauge particle, photon, exists in QED. We hope our approach be helpful to understand color confinement in QCD.

\section{References}

[1] E.P. Wigner. Phys. Rev. 40, 749(1932); M. Hillery, R.F. O'Connell, M.O. Scully and E.P. Wigner. Phys. Rep. 106 121(1984); C.W. Misner, K.C. Thorne, J.A. Wheeler, Gravitation, Freeman, San Francisco, 1970 .

[2] D. Solomon, arXiv:0710.3958, D. Solomon, Phys. Scr., 76 (2007), 64.

[3] I. Bialynicki-Birula, Decoherence and Entropy in Complex Systems, p. 287.

[4] I. Bialynicki-Birula, Opt. Comm. 179, 237(2000).

[5] C. Kiefer and A. Wipf, Annals Phys. 236, 241(1994).

[6] D.Q. Liu, Chin. Phys. 16, 1009(2007)

[7] T.D. Lee, Particle Physics and Introduction to Field Theory, Section 18.3, Columbia University Rress. 1981.

[8] J. Kijowski, G. Rudolph and M. Rudolph, arXiv:hep-th/9909113

[9] S. Weinberg, The Quantum Theory of Fields. Combridge University Press.

[10] L. Chen and K. Haller, Int.J.Mod.Phys. A14, 2745 (1999); arxiv-th/9808028. 